Recherches en didactique des langues et des cultures

Les cahiers de l'Acedle

$4 \mid 2007$

Notions en questions : Méthodologies de recherche

\title{
Didactique des langues et recherche expérimentale
}

Muriel Grosbois

\section{OpenEdition}

Journals

Édition électronique

URL : http://journals.openedition.org/rdlc/4966

DOI : $10.4000 /$ rdlc.4966

ISSN : 1958-5772

Éditeur

ACEDLE

Référence électronique

Muriel Grosbois, "Didactique des langues et recherche expérimentale ", Recherches en didactique des langues et des cultures [En ligne], 4 | 2007, mis en ligne le 21 juin 2007, consulté le 11 septembre 2019. URL : http://journals.openedition.org/rdlc/4966 ; DOI : 10.4000/rdlc.4966

Ce document a été généré automatiquement le 11 septembre 2019

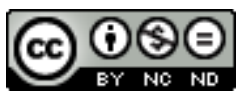

Recherches en didactique des langues et des cultures is licensed under a Creative Commons AttributionNonCommercial-NoDerivatives 4.0 International License 


\title{
Didactique des langues et recherche expérimentale
}

\author{
Muriel Grosbois
}

\section{Introduction}

1 Dans l'introduction de l'ouvrage Psychologie des apprentissages et multimédia, Crinon, Georget, Legros et Mangenot rappellent "la dichotomie souvent relevée à propos des recherches en didactique", à savoir, d'un côté, "les recherches qui visent plutôt à saisir le réel dans sa complexité comme les études de cas en psychologie et en ethnométhodologie", et, de l'autre côté, les recherches "qui utilisent une démarche hypothético-déductive, le plus souvent expérimentale" (Legros \& Crinon, $2002: 11)$. Ces points de vue divergents sont également relatés par Chapelle (Chapelle, $2003: 70$ ).

2 Les auteurs initialement cités précisent que "les critiques, de part et d'autre, sont fortes" : " manque de 'rigueur' et utilisation d'une démarche plus 'intuitive' que réellement scientifique" dans un cas; problème du contrôle strict de la situation dans l'autre cas, dans la mesure où "le 'toutes choses égales par ailleurs' [...] ne permettrait pas de rendre compte du 'réel'" (Legros \& Crinon, $2002: 11$ ), c'est-à-dire de la complexité de la situation d'apprentissage.

3 Plusieurs types de recherche sont donc envisageables en didactique des langues et on retiendra, en prenant appui sur Gagné et al. (Gagné et al., 1989), dont les travaux portent sur la didactique du français langue maternelle, qu'une recherche se caractérise par un double critère : celui de l'objectif et de la méthodologie. En ce qui concerne la recherche expérimentale, l'objectif est d'expliquer, en procédant à une expérimentation qui permet de corroborer ou de réfuter des prédictions (c'est-à-dire des hypothèses).

Simard, qui reprend la typologie de l'équipe de Gagné, précise :

S'inscrivant dans une démarche hypothético-déductive, la recherche expérimentale tente, à la lumière d'un cadre théorique, de vérifier la relation de cause à effet entre des variables en les manipulant à l'aide d'un dispositif soigneusement contrôlé (Simard, $1994:$ 487). 

recherche expérimentale, outre la validation/invalidation d'une hypothèse, sont : la mise en place d'un dispositif rigoureux, le suivi strict d'un protocole, l'identification de variables, le contrôle de la situation, ainsi que l'établissement d'une relation causale (sur la base d'un raisonnement hypothético-déductif) en vue d'aboutir à une explication.

6 Autant de notions qu'il convient d'approfondir pour définir aussi précisément que possible ce qu'est la recherche expérimentale afin d'en analyser la pertinence, ainsi que les limites, en didactique des langues. Pour cela, nous proposons de circonscrire d'abord brièvement la recherche expérimentale dans son champ d'application d'origine, puis d'analyser dans quelle mesure elle peut s'appliquer aux sciences humaines, et plus particulièrement à la didactique des langues.

\section{La recherche expérimentale: des sciences aux sciences humaines}

7 Conformément à l'évolution historique, nous tenterons ici de cerner la recherche expérimentale en sciences, puis en sciences humaines.

\subsection{La recherche expérimentale en sciences}

8 En retraçant un historique de la recherche expérimentale en sciences, nous nous sommes attachée à repérer les définitions qui en sont données ainsi que les étapes qui la caractérisent.

Dans son Introduction à l'étude de la médecine expérimentale, voici ce que Claude Bernard a écrit en 1865 :

La méthode expérimentale, en tant que méthode scientifique, repose toute entière sur la vérification expérimentale d'une hypothèse scientifique (Bernard, 1966 : 304). L'expérimentateur, [...], pose [...] son idée comme une question, comme une interprétation anticipée de la nature, plus ou moins probable, dont il déduit logiquement des conséquences qu'il confronte à chaque instant avec la réalité au moyen de l'expérience (Bernard, $1966: 60$ ).

Pour Claude Bernard, père de la démarche hypothético-déductive, c'est l'hypothèse qui est la pièce maîtresse, anticipation sur le réel qui est ensuite éprouvée au travers de l'expérience.

On retrouve là une idée déjà exprimée par Chevreul en 1856 :

Un phénomène frappe vos sens; vous l'observez avec l'intention d'en découvrir la cause, et pour cela, vous en supposez une dont vous cherchez la vérification en instituant une expérience [...]. Ce raisonnement constitue la méthode que j'appelle expérimentale, parce qu'en définitive l'expérience est le contrôle, le critérium de l'exactitude du raisonnement dans la recherche des causes ou de la vérité (Chevreul, $1856: 27-29$ ).

En d'autres termes, on émet des hypothèses et on les contrôle par la confrontation aux faits. Les hypothèses sont mises à l'épreuve de l'expérience.

13 La science cherche ainsi à "se former une représentation toujours plus fine de ce que l'on appelle "la réalité" pour reprendre François Jacob (Jacob, 1987 : 251), en établissant des relations et en mettant en évidence des mécanismes. 
Plus précisément, si l'on se réfère notamment à Jordan selon qui "Scientists articulate problems, make observations, perform experiments, propose hypotheses, build theories and test them, all the while communicating their results to colleagues" (Jordan, $2004: 83$ ), la démarche expérimentale peut se définir comme une approche construite autour des étapes suivantes.

- Un questionnement.

- Un problème à résoudre.

- La formulation d'une hypothèse (à partir de références théoriques).

- La mise à l'épreuve de l'hypothèse. Après avoir planifié les données à recueillir, ainsi que la mise en place des procédures et du suivi, les résultats sont relevés, analysés, interprétés. On compare alors les résultats attendus et les résultats observés, c'est-à-dire que l'on confronte les résultats expérimentaux aux hypothèses émises.

- Une vérification qui permet la validation.

- Une généralisation (en veillant à ce qu'elle ne dépasse pas les interprétations possibles). En fonction des résultats constatés, on élabore une relation abstraite, pour aboutir à une explication, voire à des modèles explicatifs, ou à des apports théoriques. Jordan précise :

the deductive, rationalist approach favoured causal explanations [...] - while the inductive approach gave rise to more descriptive explanations (Jordan, $2003: 46)$.

Il souligne aussi, d'une part, que dans le cas de l'induction, il est logiquement impossible de passer du particulier au général, et, d'autre part, que dans le cas de la déduction, les tests empiriques conduisent à une certitude seulement lorsque les démonstrations établissent qu'une idée est fausse, en faisant référence à Popper, sinon il est juste possible d'avancer que les hypothèses sont corroborées.

- La dernière étape est celle de la communication des résultats.

- On note également que dans la mise à l'épreuve, il importe de :

- distinguer les variables liées au phénomène étudié ;

- faire varier uniquement un facteur à la fois. De Vecchi rappelle :

Il est [donc] important de définir les différents facteurs qui interviennent dans un phénomène. Et il est tout aussi indispensable de les analyser séparément, les uns après les autres. Pour chacun, il s'agira de les faire varier, tous les autres restant à l'identique (De Vecchi, 2006 : 142-143).

De plus, comme le résume Jordan :

Experiments are observations carried out under controlled, reproducible conditions, and one of their chief functions is to allow others to carry out similar experiments in different places at different times (Jordan, $2003: 85$ ).

17 C'est-à-dire que l'expérience doit être contrôlée et reproductible. On rejoint les propos de Mialaret qui déclare :

l'essentiel est de donner des résultats qui, obtenus selon des règles précises, permettent à tous les chercheurs d'aboutir aux mêmes interprétations des résultats (Mialaret, $2004: 20$ ).

18 Les points-clés du protocole expérimental qui se dégagent ici, et qui s'ajoutent à ceux relevés dans l'introduction, sont: l'isolement des variables afin de les faire varier séparément, la reproductibilité de la situation expérimentale, et la généralisation des conclusions tirées.

Examinons maintenant dans quelle mesure cette méthode de recherche peut s'appliquer aux sciences humaines. 


\subsection{La quasi-expérimentation en sciences humaines}

expérimentale en sciences humaines et sociales :

Consider how much more fortunate the physicist is when compared to the behavioral scientist who works in field settings. The physicist can use a laboratory to create physical isolation, and he or she often works with objects that do not change over the time period of an experiment. This being so, history, maturation, and regression are not problematic, and the data from single-group pretest-posttest changes are often causally interpretable... The social scientist, on the other hand, has few of the advantages of the physicist [...], for the social scientist is trying to answer causal questions in more complex social settings where the entities being studied are clearly amenable to change for reasons that have nothing to do with the experiment (Cook \& Campbell, 1979 : 102-103).

21 Ces auteurs expriment ici qu'il est possible, en sciences physiques, d'établir un lien de cause à effet à partir d'une expérimentation car les conditions de laboratoire permettent de créer un isolement et car le chercheur travaille souvent sur des objets qui ne changent pas pendant la durée de l'expérimentation. En revanche, la situation s'avère être beaucoup plus complexe en sciences humaines et sociales.

En effet, comme nous l'avons vu précédemment, dans le cadre d'une recherche expérimentale, tout doit pouvoir être contrôlé, vérifié, reproduit, recalculé. De plus, l'expérimentation suppose une comparaison de résultats obtenus dans des situations dont on connaît les variables et dont on en fait varier UNE pour en étudier les conséquences sur les autres.

Or, en sciences humaines et sociales, et tout particulièrement en matière d'enseignement/apprentissage, les difficultés suivantes se posent.

- L'influence de facteurs extérieurs pouvant interférer lors d'une expérimentation. Certes, cette influence pourrait, dans certains cas, être limitée par une durée réduite de l'expérimentation, mais, en didactique des langues par exemple, on sait par ailleurs que l'apprentissage est un processus long et que les progrès ne sont pas toujours décelables sur un laps de temps court (les effets se manifestent plutôt à moyenne ou longue échéance).

- L'existence d'un très grand nombre de variables, et le fait que certaines ne sont pas directement observables. Comment alors agir sur une seule en maintenant toutes les autres constantes? On est ici confronté à un problème de contrôle des variables, tant le nombre de facteurs en interaction est élevé en matière d'enseignement/apprentissage. La réalité des situations pédagogiques rend ainsi difficile l'approche qui consiste à associer une cause et un effet.

- L'impossibilité de reproduire à l'identique une situation d'éducation, comme l'explique Mialaret :

les situations d'éducation, considérées sous l'angle de leur existence réelle, sont uniques, c'est-à-dire qu'elles ne se reproduisent pas à l'identique ni dans l'espace ni dans le temps. Une fois une telle situation réalisée, tous les acteurs changent par le fait qu'ils l'ont vécue, et un essai de répétition, de reproduction ne peut trouver tous les partenaires dans les mêmes conditions (Mialaret, 2004 : 10).

Par conséquent, compte tenu de la complexité des situations d'enseignement/ apprentissage due à la réalité multifactorielle qui les caractérise, ce sont plutôt des plans quasi expérimentaux qui sont mis en œuvre en sciences humaines, lorsque cette 
démarche est retenue. Ainsi, on choisira de ne considérer qu'un nombre limité de variables et de recueillir des données aussi explicites que possible. On constituera des groupes quasi équivalents pour faire subir à l'un d'eux l'action expérimentale et comparer ensuite les résultats obtenus avec le maximum d'objectivité. Intéressons-nous maintenant plus spécifiquement à la recherche (quasi) expérimentale en didactique des langues, ou, pourrait-on dire, au choix du chercheur de se positionner plutôt du côté de la recherche (quasi) expérimentale.

\section{Didactique des langues et recherche (quasi) expérimentale}

Sachant que l'objet de recherche en didactique des langues relève d'une interaction de plusieurs facteurs, différents types de recherche sont envisageables et ils sont souvent perçus comme dichotomiques, ainsi que nous l'avons souligné en introduction. Le choix méthodologique du chercheur dépend donc son positionnement. Par conséquent, il importe qu'il le définisse clairement.

\subsection{Positionnement du chercheur}

Narcy-Combes rappelle les paramètres qui entrent en jeu en matière de positionnement, au moyen des questions suivantes.

- La recherche est-elle synthétique ou analytique?

- Les objectifs sont-ils heuristiques ou déductifs?

- Quel est le contrôle ou le degré de contrôle du contexte de la recherche?

- De quel ordre seront les données et le recueil des données? (Narcy-Combes, 2005 : 100)

L'auteur représente ensuite les paramètres sous la forme de continua (figure 1) et précise :

En fonction de la façon dont une recherche permet de gérer les paramètres, on peut en déterminer le type (Narcy-Combes, $2005: 103$ ).

Figure 1 (Narcy-Combes, $2005:$ 103)

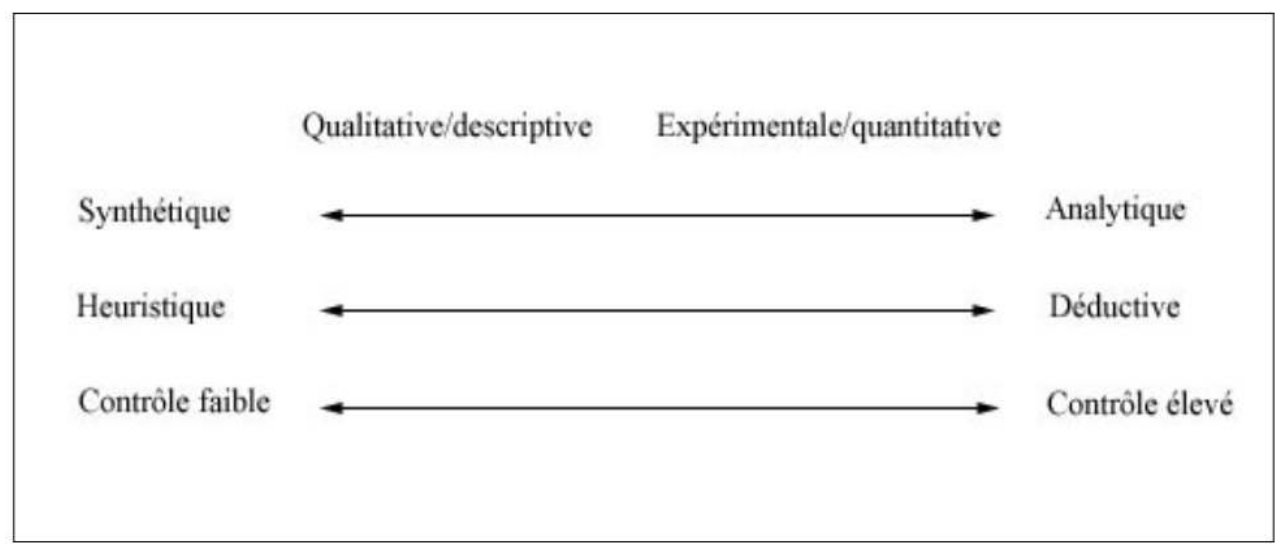

En situant la recherche en didactique des langues sur la base de ces continua, comment se définit alors la recherche (quasi) expérimentale? 


\subsection{Choix de la recherche (quasi) expérimentale}

Narcy-Combes explique :

Sur cet ensemble de continua, la recherche expérimentale se situera du côté analytique, déductif, avec un contrôle élevé et des données explicites (NarcyCombes, 2005 :103).

On rejoint ici la définition que nous avons précédemment donnée de la recherche expérimentale qui consiste à étudier une composante d'un phénomène global, selon la démarche descendante dont nous avons décrit les différentes étapes au paragraphe 2.1.

Et Narcy-Combes ajoute, en s'appuyant sur le schéma qui suit, emprunté à Juan (figure 2), que la recherche expérimentale peut faire appel à :

- des entretiens et/ou une recherche-action, dans une approche compréhensive,

- des questionnaires établis en fonction de théories et/ou des expériences en

laboratoire, dans une approche explicative (Narcy-Combes, 2005 : 104).

Figure 2 (Juan, 1999 : 13)

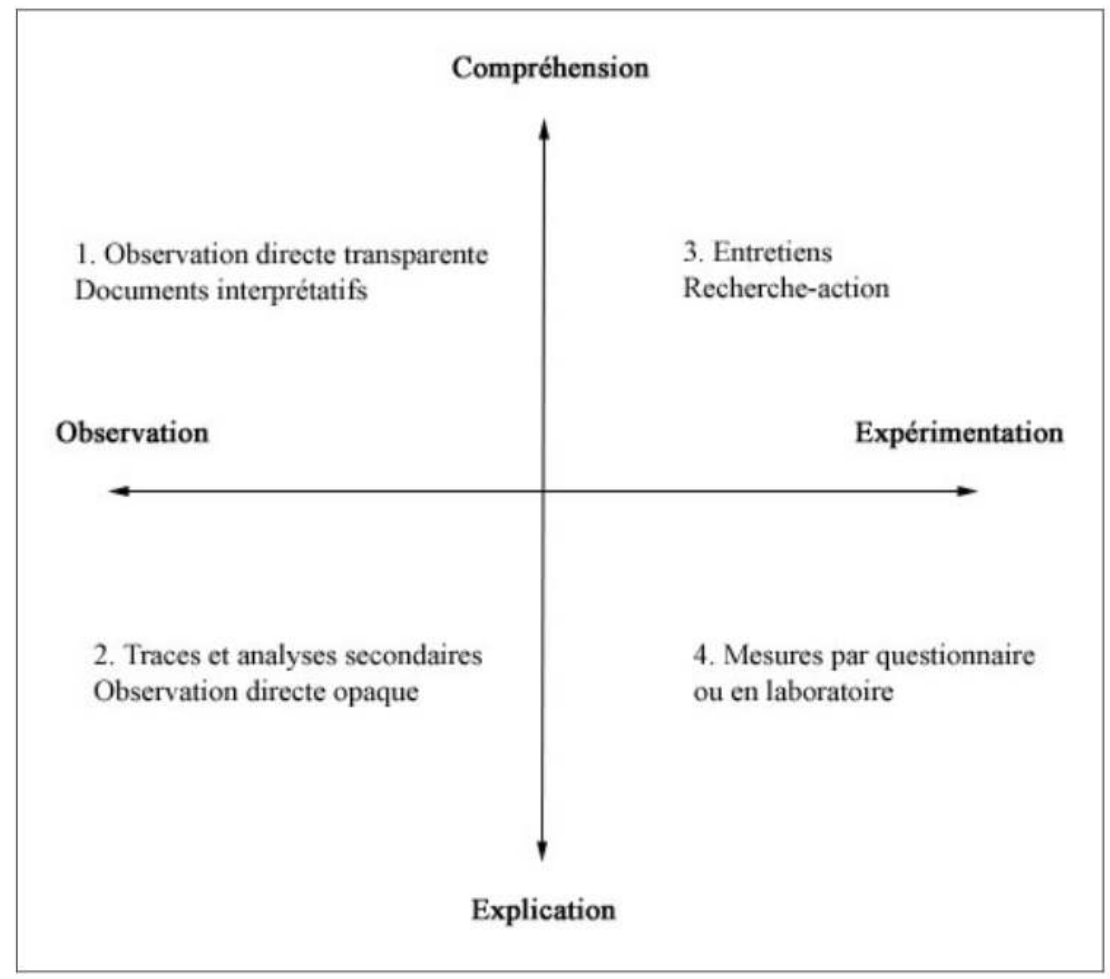

Cela permet de dépasser la vision dichotomique que nous avons initialement relevée et de considérer, qu'en didactique des langues, il existe une complémentarité entre les différentes approches, à condition que les choix méthodologiques soient logiques, et que l'emploi des outils soit cohérent.

\section{Exemples en didactique des langues}

Souhaitant consacrer la troisième partie de cet exposé à des exemples de recherches (quasi) expérimentale en didactique des langues, on s'aperçoit qu'ils s'inscrivent soit dans 
un cadre (quasi) expérimental, soit à un croisement méthodologique (dans la mesure où ils font pour partie seulement appel à l'expérimental). Cela illustre les propos qui viennent d'être tenus sur la complémentarité des approches dans un domaine aussi complexe que celui de l'enseignement/apprentissage d'une langue 2 (L2).

\subsection{Exemples de recherche (quasi) expérimentale}

\subsubsection{Expérimentation sur l'effet du double codage synchrone de l'accentuation en} L2

Cette étude a été menée par Stenton, Péchou, Vaillant-Sirdey et Tricot, et présentée lors du premier colloque international de didactique cognitive à Toulouse les 26-28 janvier 2005 (Stenton et al., 2005).

Précisons tout d'abord que leurs travaux portent sur la difficulté qu'ont les apprenants francophones à encoder l'information relative à l'accent tonique en anglais et par conséquent à le mémoriser, le produire ou le reproduire.

Le projet vise à développer un système auteur qui permet de mettre en exergue auditivement et visuellement la syllabe accentuée d'un mot afin de focaliser l'attention de l'apprenant sur ce point délicat qu'est l'accentuation en anglais.

Examinons la démarche expérimentale mise en œuvre par cette équipe de chercheurs.

Leurs hypothèses sont que :

- le double codage de la syllabe accentuée améliore les performances des novices ;

- il n'améliore pas ou il détériore les performances des experts ;

- ce double effet s'observe avec les modalités de restitution écrites et orales;

- les performances sont meilleures pour une tâche de rappel écrit que pour une tâche de rappel oral.

Ils ont testé ces hypothèses empiriquement de la façon suivante.

Trois groupes de participants ont été créés : les experts/ les intermédiaires/les novices.

41

Des listes de 10 mots anglais leur ont été présentées, chacune selon deux modalités ${ }^{1}$ différentes :

- codage audio seul ;

- double codage (audio et visuel);

Pour ce qui relève du protocole et de la mesure, on note que :

- chaque groupe est divisé en sous-groupes ;

- chaque sous-groupe travaille sur une seule modalité de présentation des mots ;

- les modalités de restitution sont des épreuves de rappel écrit et oral. Dans le cas du rappel écrit, l'étudiant entend un mot et doit cocher sur une feuille reproduisant la liste des 10 mots, la syllabe accentuée en face du mot correspondant. Dans le cas du rappel oral, l'étudiant entend un mot et doit le prononcer. Les réponses sont codées de façon binaire : accent tonique placé correctement $=1$, accent tonique placé incorrectement $=0$.

43 Les chercheurs analysent ensuite les résultats obtenus, ce qui leur permet, dans un second temps, de valider ou non leurs hypothèses de départ, qui ont été mises à l'épreuve du test. 

clés suivants.

- La recherche porte ici sur un domaine bien circonscrit.

- Dans le protocole, les groupes de niveau sont constitués en fonction de l'objectif de la recherche, et on ne fait varier qu'une seule variable à la fois (le codage visuel). On observe ensuite ce qui se passe pour chacun des groupes de niveaux différents.

- La mise à l'épreuve des hypothèses permet un retour sur ces hypothèses (qui sont alors validées ou non).

- Le cadre expérimental permet d'obtenir une finesse de l'observation permise par la situation de laboratoire. Les conclusions ne sont pas contextualisées et ont donc une valeur générale. En revanche, on ne se situe pas dans le cadre d'une pratique authentique, on n'apporte donc pas d'informations supplémentaires sur la situation d'apprentissage au sens large. Toutefois, l'expérimentation n'est pas dépourvue de retombées pratiques (même si elles sont indirectes). En effet, le système pourrait, à terme, permettre aux apprenants francophones de se faire mieux comprendre de locuteurs natifs, et on sait combien cela s'avère être nécessaire si l'on considère ne serait-ce que les effets de la nativisation phonologique qui opère lors de l'apprentissage de la L2, et qu'il convient de contrecarrer. Rappelons que la nativisation, concept développé par Andersen (Andersen, 1983), consiste à traiter toute nouvelle donnée langagière en fonction de critères déjà intériorisés, dont ceux de la langue 1 (L1), les autres langues déjà acquises ainsi que les représentations que les apprenants se font de la L2 interférant également.

\subsubsection{Expérimentation sur l'effet du sous-titrage dans l'acquisition de la L2}

Cette recherche a été menée par Borrás \& Lafayette en 1994 (Borrás \& Lafayette, 1994). Elle est catégorisée par Chapelle comme une quasi-expérimentation (Chapelle, $2003:$ 91). avoir accès aux sous-titres lorsqu'ils sont confrontés à des difficultés de compréhension orale. Les autres visualisent la même vidéo dans les mêmes conditions, mais ne peuvent pas avoir accès aux sous-titres.

production orale a été mesurée en demandant aux apprenants des deux groupes de transmettre des informations sur le contenu vidéo. Ils ont été évalués sur la base de quatre critères (l'efficacité, la précision, l'organisation et la fluidité). Les résultats se sont avérés supérieurs dans le cas où les sous-titres étaient accessibles.

51 La conclusion qui en est tirée est que, dans le cadre d'un travail à partir d'un support vidéo authentique, la possibilité de voir et de contrôler les sous-titres améliore l'expression en L2 (en plus de la compréhension).

52 Les points-clés soulignés pour l'exemple précédent valent également pour celui-ci. On s'aperçoit en effet que, dans ce protocole quasi expérimental, on agit sur une variable (l'accès possible aux sous-titres), puis on mesure et compare les résultats obtenus par les 
apprenants des deux groupes (groupes que l'on peut considérer comme quasi équivalents).

\subsection{Exemples de croisement méthodologique}

\subsubsection{Recherche sur l'influence de l'outil d'écriture}

étude, conduite par Snyder en 1994 (Snyder, 1994), vise à analyser l'influence de l'outil d'écriture (traitement de texte ou crayon), sur la qualité des textes produits par les apprenants, sur les processus d'écriture, ainsi que sur les contextes d'enseignement/ apprentissage.

L'expérience a été menée pendant une année scolaire dans deux classes de huitième année dont les apprenants avaient la même enseignante et pratiquaient les mêmes activités d'écriture.

Les élèves de la première classe ont utilisé uniquement le traitement de texte, ceux de la deuxième classe ont rédigé uniquement au crayon. Les textes produits (narratifs, argumentatifs et informatifs) ont été évalués en aveugle par des évaluateurs extérieurs. Et l'auteur précise que la méthode quasi expérimentale avec (pré-test et post-test) a été complétée par des questionnaires, des observations et des entretiens.

Les conclusions de cette recherche sont que :

- la qualité des textes (surtout argumentatifs et informatifs) produits par le groupe-classe utilisant le traitement de texte, est supérieure à celle des textes produits par le groupeclasse utilisant le papier/crayon ;

- les résultats ne laissent pas apparaître de différence dans les stratégies de planification et de révision des apprenants ;

- des différences de contexte pédagogique ont été relevées entre les deux classes. Par exemple, la classe utilisant le crayon a tendance à être centrée sur l'enseignante, alors que les élèves utilisant le traitement de texte interagissent plus entre eux pour l'accomplissement de la tâche et explorent davantage les diverses possibilités d'écriture.

On constate dans cet exemple un recours au quantitatif ET au qualitatif.

De ce fait, on obtient aussi des informations sur la situation d'apprentissage (contrairement aux exemples précédents qui font appel à une situation de laboratoire, et non de terrain).

Plus précisément, la variété des outils de recueil de données utilisés, en adéquation avec l'objectif de la recherche, a permis de mettre en relation l'influence de l'outil d'écriture sur les textes produits et les contextes d'enseignement/apprentissage, puisque l'une des conclusions de l'auteur est que le traitement de texte a permis d'installer de nouvelles modalités de travail, de type collaboratif et coopératif, qui favorisent l'apprentissage de la production de texte.

60 On retrouve là l'intérêt de la complémentarité des approches que nous avons présentée au paragraphe 3.2., et on rejoint le point de vue de l'équipe de Gagné, résumé ainsi par Simard:

une recherche peut être à la fois quantitative et qualitative si, par exemple, en plus d'utiliser un plan expérimental et des analyses statistiques les chercheurs recourent à des techniques qualitatives telles que l'entrevue ou l'étude de cas pour mieux comprendre ce qui s'est passé chez les élèves durant le traitement pédagogique mis à l'épreuve (Simard , 1994 : 488). 


\subsubsection{Incursion en recherche quasi expérimentale dans le cadre d'une recherche- action}

Cet exemple est celui d'une recherche que nous avons menée (Grosbois, 2006) et qui porte sur un projet collectif de création multimédia entre des stagiaires professeurs des écoles de l'IUfM de Paris et des étudiants du King's College de Londres. Ces partenaires ont communiqué en anglais par courriel en vue de la création commune d'une ressource numérique. Nous avons fait l'hypothèse qu'un tel projet est susceptible d'opérer comme levier d'apprentissage de l'anglais (L2) pour les stagiaires de l'IUFM.

Il s'agit d'une recherche de type recherche-action qui nous a permis de mesurer la portée et les limites du dispositif mis en place en regard de l'objectif fixé, et de déboucher sur de nouvelles propositions de formation (conformément au cycle de la recherche-action). Toutefois, cette recherche intègre un volet qui est de l'ordre de la quasi-expérimentation pour ce qui relève de l'étude du phénomène de nativisation phonologique. L'étude de ce phénomène se justifie par le fait que l'une des macro-tâches du scénario de formation consiste, pour les stagiaires, à partager oralement avec le groupe-classe le contenu des courriels qu'ils ont chacun reçus de leurs partenaires du King's College. De ce fait, l'input authentique servant de support à leur production orale est exclusivement de l'ordre de l'écrit, il est donc dépourvu de dimension phonologique. Or, les références théoriques soulignent que l'apprentissage de la L2 souffre de problèmes d'interférence de la L1 (entre autres), liés au phénomène de nativisation. De plus, Shen et Forster (Shen \& Forster, 1999) n'excluent pas le fait que l'activation phonologique soit automatique lors d'une tâche de production orale émanant d'un support écrit. La question que nous avons alors posée est celle du plan phonologique mis en place par les apprenants exécutant une macro-tâche de production orale reposant sur un input écrit. N'y a-t-il pas là un risque de nativisation plus grand que lorsque l'input est de l'ordre de l'oral? Afin de valider ou non cette hypothèse, nous nous sommes livrée à une expérimentation qui a consisté à comparer les productions orales des stagiaires à partir de supports écrits (les courriels qu'ils avaient reçus de leurs correspondants anglophones), et les productions orales des stagiaires à partir de supports oraux (des enregistrements sur cassettes).

Dans un premier temps, nous avons indexé, pour l'ensemble des stagiaires, les items de chacune de leurs productions orales ainsi que les items des supports correspondants (soit l'enregistrement sur cassette, soit le courriel), au moyen d'un concordancier. Dans un second temps, le concordancier a permis de répertorier, sous forme de liste, les items appartenant à la fois à une production orale et au support correspondant.

Ensuite, nous avons sélectionné, pour chaque liste ainsi établie, les items qui présentaient un écart de prononciation par rapport aux objectifs fixés.

Nous avons conclu que le pourcentage d'items repris (du support) qui présentent un écart de prononciation est supérieur lorsque le support est de l'ordre de l'écrit.

Nous avons ensuite procédé à une analyse phonétique des items présentant un écart de prononciation. Il s'est avéré que les difficultés de prononciation rencontrées relevaient de l'influence de la L1 des stagiaires. Nous avons ainsi pu établir un lien entre les écarts de prononciation et le phénomène de nativisation (celui exercé par la L1, à laquelle nous nous sommes limitée) et déduire que la nativisation opère davantage lorsque la production orale relève d'un input écrit, que lorsque la production orale relève d'un input oral, et ainsi confirmer notre hypothèse. 
Dans cette recherche, qualitatif et quantitatif ont ainsi été combinés, ce qui a permis d'enrichir note étude et d'apporter des informations à la fois sur la situation d'apprentissage (pour la partie recherche-action) mais aussi sur un point très spécifique en matière d'apprentissage de la L2, celui du phénomène de nativisation (au moyen d'une quasi-expérimentation).

\section{Conclusion}

Dans un domaine aussi complexe que celui de la didactique des langues, plusieurs types de recherche sont envisageables, comme nous l'avons signalé en introduction. Mais plutôt que de s'exclure, il s'avère qu'ils se complètent dans la mesure où ils renseignent différemment. En effet, la recherche (quasi) expérimentale permet d'accéder à des informations sur le rôle d'un facteur particulier, et pour cela, elle fige en quelque sorte la réalité. Elle ne permet donc pas de saisir la complexité et la dynamique d'une situation d'enseignement/apprentissage dans son ensemble, contrairement aux recherches qualitatives.

Certes au début des années 70, c'est la recherche expérimentale qui était prônée en didactique des langues. On se situait essentiellement dans un cadre qui se voulait résolument scientifique et qui consistait à formuler des hypothèses et à les mettre à l'épreuve afin d'aller au-delà de l'empirisme des praticiens. On se soumettait alors aux contraintes du protocole expérimental: isolement de variables, comparaison, quantification, extériorité de l'observation, formalisation. A cette méthode de recherche s'ajoutent aujourd'hui d'autres approches qui ne produisent pas une explication à valeur généralisante, mais qui aident à comprendre la situation d'enseignement/apprentissage au sens large.

En fait, les options épistémologiques sont fonction de l'objectif de la recherche et de la posture du chercheur. Les méthodologies employées diffèrent et se complètent, comme l'expriment Chapelle et Jordan :

I have suggested that research methods need to be tied to the research questions that are posed by the researcher [...]. From this perspective, the focus is not a single theoretical orientation but a quality of the CALL task (e.g. language learning potential) for which the research seeks evidence (Chapelle, 2003:80).

SLA research needs a multi-method approach (Jordan, $2004: 116)$.

71 Il conviendrait donc plutôt de considérer la recherche en didactique des langues en termes de pluralité d'approches, voire de croisements méthodologiques, afin d'inclure un regard vers le social, pour produire, au final, un savoir au service de la pratique.

\section{BIBLIOGRAPHIE}

Andersen, R. (1983). Pidginization and Creolization as Language Acquisition. Rowley, MA : Newbury House. 
Bernard, C. (1865). Introduction à l'étude de la médecine expérimentale. Paris : Garnier-Flammarion, 1966.

Borrás, I. \& Lafayette, R. C. (1994). "Effects of Multimedia Courseware Subtitling on the Speaking Performance of College Students of French". The Modern Language Journal, vol. 78, $n^{\circ} 1$. pp 61-75.

Chapelle, C. (2003). English Language Learning and Technology. Amsterdam/Philadelphia : John Benjamins Publishing Company.

Chevreul, E. (1856). Lettres adressées à M. Villemain sur la méthode en général et sur la définition du mot FAIT : relativement aux sciences, aux lettres, aux beaux-arts, etc., etc. Paris : Garnier Frères.

Cook, T. \& Campbell, D. (1979). Quasi-Experimentation. Design and Analysis Issues for Field Settings. Houghton Mifflin Company : Boston.

De Vecchi, G. (2006). Enseigner l'expérimental en classe. Paris : Hachette.

Gagné, G., Lazure, R., Sprenger-Charolles, L. \& Ropé, F. (1989). Recherches en didactique et acquisition du français langue maternelle. Tome 1 : Cadre conceptuel, thésaurus et lexique des mots-clés. Montréal : De Boeck-Université, Editions universitaires, Institut national de Recherche pédagogique.

Grosbois, M. (2006). Projet collectif de création d'une ressource numérique comme levier d'apprentissage de l'anglais. Thèse de doctorat. Université Paris III.

Jacob, F. (1987). La Statue intérieure. Paris : Seuil.

Jordan, G. (2004). Theory Construction in Second Language Acquisition. Amsterdam/Philadelphia : John Benjamins Publishing Company.

Juan, S. (1999). Méthodes de recherche en sciences sociohumaines. Paris : PUF.

Legros, D. \& Crinon, J. (2002). Psychologie des apprentissages et multimédia. Paris : Armand Colin.

Mialaret, G. (2004). Les méthodes de recherche en science de l'éducation. Paris : Presses Universitaires de France.

Narcy-Combes, J.-P. (2005). Didactique des langues et TIC : vers une recherche-action responsable. Paris : Ophrys.

Shen, D. \& Forster, K. (1999). "Masked Phonological Priming in Reading Chinese Words Depends on the Task". Language and Cognitive Processes, vol. 14, n 5. pp. 429-459.

Simard, C. (1994). "La didactique du français langue maternelle : analyse d'une recherche bibliographique fondamentale". Revue canadienne de l'éducation, vol. 19, n 4. pp. 481-489.

Snyder, I. A. (1994). "Writing with word processors : The computer's influence on the classroom context". Journal of Curriculum Studies, vol. 29, n² 2. pp. 143-162.

Stenton, A., Péchou, A., Vaillant-Sirdey, C. \& Tricot, A. (2005). "Effet du double codage synchrone de l'accentuation en L2 selon des modalités de restitution de l'apprenant". Premier colloque international de didactique cognitive, Toulouse. Disponible en ligne. http://perso.orange.fr/ andretricot/AndreTricot.html (lien valide à la date de publication)

\section{NOTES}

1. Précisons que le protocole a intégré trois modalités, mais nous n'en avons mentionné que deux ici. 
2. Ce résumé se limite aux points les plus pertinents pour la présentation.

\section{RÉSUMÉS}

Cet article traite de la pertinence, mais aussi des limites de la recherche expérimentale en didactique des langues.

Après avoir circonscrit les points-clés de la recherche expérimentale en sciences, le recours à ce type de recherche en sciences humaines fait ensuite l'objet d'une analyse. Puis, la réflexion se centre sur la didactique des langues et est illustrée par plusieurs exemples. A l'issue de l'argumentation, la question suivante est posée: n'est-ce pas plutôt en termes de pluralité d'approches qu'il conviendrait d'envisager la recherche dans un domaine aussi complexe que celui de la didactique des langues?

This article deals with both the interest and the limits of experimental research in the field of language 2 (L2) learning and teaching.

After defining the key factors of experimental research in science, its application to human sciences will be analysed. Its use for L2 research will then be tackled and illustrated by several examples. Given the complexity of language learning and teaching, the need for multifaceted approaches will finally be discussed.

\section{INDEX}

Mots-clés : hypothèse, expérience, explication, variable

Keywords : hypothesis, experiment, explanation, variable

\section{AUTEUR}

\section{MURIEL GROSBOIS}

Muriel Grosbois enseigne à l'IUFM de Paris et a soutenu sa thèse à Paris III en 2006 sous la direction de Françoise Demaizière. Sa recherche porte sur la création multimédia, en présentiel et à distance, comme levier d'apprentissage de l'anglais. Son laboratoire de rattachement est le Diltec, université Paris 3, groupe "Tâches et dispositifs".

Courriel : muriel.grosbois[at]paris.iufm.fr

Adresse : IUFM de Paris, 10 rue Molitor, 75016 Paris, France. 